\title{
CONVERGENCE ESTIMATES OF POD-GALERKIN METHODS FOR PARABOLIC PROBLEMS
}

\author{
Thibault Henri \\ INSA de Rennes, IRMAR \\ CS 14315, 35043 Rennes Cedex \\ Thibault.Henri@insa-rennes.fr \\ Jean-Pierre Yvon \\ INSA de Rennes, IRMAR \\ Jean-Pierre.Yvon@insa-rennes.fr
}

Abstract Proper orthogonal decomposition (POD) is a Galerkin method which has been introduced in a fluids mechanics context. It is also known as Karhunen-Loeve decomposition and principal component analysis. The idea of POD consists in using a priori known information on the solution $u$ of PDE, for example snapshots $u_{i}=u\left(t_{i}\right)$, to determine a set of functions which are the eigenfunctions of an Hilbert-Schmidt operator. This basis can be used to solve the PDE with a smaller amount of computations. Convergence estimates have been proved recently in the parabolic case starting from a particular discretization scheme [7]. Moreover it has been proved that the method converges independently from the scheme [6]. We consider the case of a linear parabolic equation. We give a first convergence estimate in a case where $u$ is regular. However classical POD does not look satisfactory and an improvement consists in considering a POD which takes into account the derivative of $u$. We will also present some insights into the control of the approximation by introducing what will be called a good order of approximation.

Keywords: Proper orthogonal decomposition, Karhunen-Löve decomposition, model reduction. 


\section{Principle of Proper Orthogonal Decomposition (POD)}

\subsection{Proper Orthogonal Decomposition of a Function $u \in L^{2}(0, T ; X)$}

Let $X$ be a real separable Hilbert space endowed with the scalar product $(., .)_{X}$, let $T>0$ be a positive real and let $u \in L^{2}(0, T ; X)$ be a (class of) function depending on time $t \in[0, T]$ with values in $X$. We define the POD operator $K(u): X \rightarrow X$ :

$$
K(u): \varphi \longmapsto \frac{1}{T} \int_{0}^{T}(u(t), \varphi)_{X} u(t) d t .
$$

We consider the kernel $\widetilde{k}(s, t)=\frac{1}{T}(u(s), u(t))_{X}$ and we define the auxiliary POD operator $\widetilde{K}(u): L^{2}(0, T) \rightarrow L^{2}(0, T)$ :

$$
\widetilde{K}(u): v \longmapsto \int_{0}^{T} v(s) \widetilde{k}(s, .) d s .
$$

By concern of clarity, we denote $K=K(u)$ and $\widetilde{K}=\widetilde{K}(u)$. The operators $K$ and $\widetilde{K}$ are self-adjoint semi-definite. Moreover $\widetilde{K}$ is a HilbertSchmidt operator since the kernel $\widetilde{k}$ is $L^{2}\left([0, T]^{2}\right)$. We can therefore index the eigenvalues of $\widetilde{K}$ in a non-increasing sequence: $\widetilde{\lambda}_{1} \geq \widetilde{\lambda}_{2} \geq$ $\cdots \geq \tilde{\lambda}_{k} \geq \cdots \geq 0$. We assume that $u$ is not the null function so that the spectrum of $\widetilde{K}$ is not zero.

LEMMA 1 The operators $K$ and $\widetilde{K}$ have the same eigenvalues with same multiplicity.

Proof. Let $\tilde{\lambda}$ be an eigenvalue of the operator $\widetilde{K}$ with multiplicity $r \geq$ 1. We consider a set $\left(v_{k}\right)_{1 \leq k \leq r}$ of orthonormal eigenvectors in $L^{2}(0, T)$ and we define the set $\left(\psi_{k}\right)_{1 \leq k \leq r}$ by posing $\psi_{k}=1 / \sqrt{\widetilde{\lambda} T}\left(u, v_{k}\right)_{L^{2}(0, T)}$. Then the set $\left(\psi_{k}\right)_{1 \leq k \leq r}$ is a set of orthonormal eigenvectors of the operator $K$ in $X$ for the eigenvalue $\tilde{\lambda}$. Conversely, if $\lambda$ is an eigenvalue of the operator $K$ and if $\left(\psi_{k}\right)_{1 \leq k \leq r}$ is a set of orthonormal eigenvectors of $K$ in $X$, the set $\left(v_{k}\right)_{1 \leq k \leq r}$ defined by $v_{k}=1 / \sqrt{\lambda T}\left(u, \psi_{k}\right)_{X}$ is a set of orthonormal eigenvectors of $\widetilde{K}$ in $L^{2}(0, T)$ for the eigenvalue $\lambda$.

DEFINITION 2 The non zero eigenvalues of the operator $K$, indexed in a non increasing order, are called the POD eigenvalues associated with the function $u$. A set $\left(\psi_{k}\right)_{k \geq 1}$ of orthonormal eigenvectors of $K$ in $X$ corresponding to these eigenvalues is called a set of $P O D$ eigenvectors associated with the function $u$. 
Remark. The POD eigenvectors depend on the space $X$. If the fonction $u$ is in $L^{2}\left(0, T ; X_{1}\right) \cap L^{2}\left(0, T ; X_{2}\right)$, the POD eigenvectors in $X_{1}$ differ from the POD eigenvectors in $X_{2}$.

Remark. We have not uniquess of the POD eigenvectors, although we have uniquess of the eigenspaces of the operator $K$. We define the approximated subspaces $Y_{\ell}=\operatorname{span}\left(\psi_{1}, \cdots, \psi_{\ell}\right)$ which do not depend of the POD vectors in the case when $\lambda_{\ell}>\lambda_{\ell+1}$ and we denote $Y=$ $\overline{\operatorname{span}\left(\psi_{k}\right)_{k \geq 1}} X$ the space spanned by the POD eigenvectors in $X$.

Remark. The POD eigenvectors correspond to eigenvalues which are indexed in a non increasing order: the order of indexation is significant.

Example. The method of snapshots. Let us consider real numbers $\sigma>0$ and $0=t_{0}<\cdots<t_{N}=T$ such that $t_{i+1}-t_{i}=\sigma$ for $i=0, \cdots, N-1$. Let $u$ be a piecewise constant function defined by posing $u(t)=u_{i+1} \in X$ for $\left.\left.t \in\right] t_{i}, t_{i+1}\right]$. The values $\left(u_{i}\right)_{1 \leq i \leq N}$ are called snapshots. The proper orthogonal decomposition of $u$ corresponds to the method of snapshots. For any $\varphi \in X$, we have: $K(u) \varphi=$ $1 / N \sum_{i=1}^{N}\left(u_{i}, \varphi\right)_{X} u_{i}$. The method of snapshots consists in considering the correlation matrix $K_{\text {cor }}$ defined by:

$$
K_{\text {cor }}=\left(\frac{1}{N}\left(u_{i}, u_{j}\right)_{X}\right)_{1 \leq i, j \leq N} .
$$

Then we denote $v_{k}=\left(v_{i, k}\right)_{1 \leq i \leq N} \in \mathbf{R}^{N}$, for $k=1, \cdots, N$, a set of orthonormal eigenvectors of the matrix $K_{\text {cor }}$. Orthonormal eigenvectors of the operator $K(u)$ are given by posing: $\psi_{k}=1 / \sqrt{N \lambda_{k}} \sum_{i=1}^{N} v_{i, k} u_{i}$. In practice, we compute the proper orthogonal decomposition of a function $u$ by approaching $u$ with a piecewise constant function. The method of snapshots allows us to compute the proper orthogonal decomposition of this piecewise constant function.

Let us mention at last two characterizations of the POD eigenvectors.

THEOREM 3 Let $\left(\psi_{k}\right)_{k \geq 1}$ be a set of POD eigenvectors associated with $u$. Then for any $\ell \geq 0$, the vector $\psi_{\ell+1}$ satisfies:

$$
\frac{1}{T} \int_{0}^{T} \frac{\left(u(t), \psi_{\ell+1}\right)_{X}^{2}}{\left(\psi_{\ell+1}, \psi_{\ell+1}\right)_{X}} d t=\max _{\varphi \in \operatorname{span}\left(\psi_{1}, \cdots, \psi_{\ell}\right)^{\perp} \backslash\{0\}} \frac{1}{T} \int_{0}^{T} \frac{(u(t), \varphi)_{X}^{2}}{(\varphi, \varphi)_{X}} d t .
$$

Conversely, if $\left(\psi_{k}\right)_{k \geq 1}$ is a set of orthonormal vectors in $X$ which satisfy equality (4), then $\left(\bar{\psi}_{k}\right)_{k \geq 1}$ is a set of $P O D$ eigenvectors associated with $u$.

Remark. In case when the function $u$ is piecewise constant, we still call the values of $u$ snapshots. The previous proposition characterizes the 
POD eigenvectors as the best correlated to the snapshots in a quadratic mean sense.

THEOREM 4 Let $\left(\psi_{k}\right)_{k \geq 1}$ be a set of POD eigenvectors associated with $u$. For any integer $\ell \geq 0$ and for any orthonormal set $\left(\varphi_{k}\right)_{k>1}$ in $X$, we have the following inequality:

$$
\int_{0}^{T}\left\|u(t)-\sum_{k=1}^{\ell}\left(u(t), \psi_{k}\right)_{X} \psi_{k}\right\|_{X}^{2} d t \leq \int_{0}^{T}\left\|u(t)-\sum_{k=1}^{\ell}\left(u(t), \varphi_{k}\right)_{X} \varphi_{k}\right\|_{X}^{2} d t
$$

Moreover we have:

$$
\frac{1}{T} \int_{0}^{T}\left\|u(t)-\sum_{k=1}^{\ell}\left(u(t), \psi_{k}\right)_{X} \psi_{k}\right\|_{X}^{2} d t=\sum_{k=\ell+1}^{\infty} \lambda_{k} \underset{\ell \rightarrow \infty}{\longrightarrow} 0 .
$$

In particular for $\ell=0$, the sum of the $P O D$ eigenvalues is equal to the energy of $u$. Conversely, if $\left(\psi_{k}\right)_{k \geq 1}$ is a set of orthonormal vectors in $X$ which satisfy equations (5) and (6), then $\left(\psi_{k}\right)_{k \geq 1}$ is a set of POD eigenvectors associated with $u$.

\subsection{Proper Orthogonal Decomposition of a Set of Functions}

Let $n \in \mathbf{N}^{*}$ be a positive integer and let $\left(u_{i}\right)_{1<i<n}$ be a set of (class of) functions in $L^{2}(0, T ; X)$. We define the POD operator $K: X \rightarrow X$ by posing:

$$
K: \varphi \longmapsto \sum_{i=1}^{n} \frac{1}{T} \int_{0}^{T}\left(u_{i}(t), \varphi\right)_{X} u_{i}(t) d t .
$$

DEFINITION 5 The non zero eigenvalues of the operator $K$, indexed in a non increasing order, are called the $P O D$ eigenvalues associated with the set $\left(u_{i}\right)_{1 \leq i \leq n}$. A set $\left(\psi_{k}\right)_{k \geq 1}$ of orthonormal eigenvectors of $K$ in $X$ corresponding to these eigenvalues is called a set of POD eigenvectors associated with $\left(u_{i}\right)_{1 \leq i \leq n}$.

The theorems analogous to theorems 3 and 4 hold.

Example. Let us consider a function $u \in L^{2}(0, T ; X)$. If the time derivative $d u / d t$ is in $L^{2}(0, T ; X)$, we can consider the proper orthogonal decomposition associated with $(u, d u / d t)$.

\section{Problem Formulation}

Let $V$ and $H$ be real separable Hilbert spaces. We assume that the embedding $V \subset H$ is dense continuous. So there exists a constant $\alpha>0$ 
such that $\|\cdot\|_{H} \leq \alpha\left\|_{.}\right\|_{V}$. We identify the space $H$ with the dual space $H^{\prime}$. Then the space $H=H^{\prime}$ is identified with a dense subspace of the dual $V^{\prime}$ of the space $V$, with continuous embedding.

Let $a: V \times V \rightarrow \mathbf{R}$ a bilinear continuous elliptic form. So there exist real numbers $\beta, \kappa>0$ such that for any $\varphi, \psi \in V$ we have $|a(\varphi, \psi)| \leq$ $\beta\|\varphi\|_{V}\|\psi\|_{V}$ and $\kappa\|\varphi\|_{V}^{2} \leq a(\varphi, \varphi)$. Let $\phi \in H$ and $f \in L^{2}\left(0, T ; V^{\prime}\right)$, with $T>0$. We consider the following parabolic problem:

$$
(\mathcal{P})\left\{\begin{aligned}
\frac{d}{d t}(u(t), \varphi)_{H}+a(u(t), \varphi) & =\langle f(t), \varphi\rangle_{V^{\prime} \times V} & & t \in[0, T], \varphi \in V, \\
(u(0), \varphi)_{H} & =(\phi, \varphi)_{H} & & \varphi \in V,
\end{aligned}\right.
$$

where $(., .)_{H}$ is the scalar product in $H$ and $\langle., .\rangle_{V^{\prime} \times V}$ is the duality $V^{\prime}, V$. We denote $W(0, T ; V)=\left\{u \in L^{2}(0, T ; V) \mid d u / d t \in L^{2}\left(0, T ; V^{\prime}\right)\right\}$ the space of (class of) functions in $L^{2}(0, T ; V)$ the time derivative of which is in $L^{2}\left(0, T ; V^{\prime}\right)$. We denote $C(0, T ; H)$ the space of continuous functions with values in $H$. The space $W(0, T ; V)$ is identified with a subspace of $C(0, T ; H)$ in the following sense: any (class of) function in $W(0, T ; V)$ admits a continuous representative with values in $H$. We have the following result ([4], theorems 1 and 2, p. 619-620):

TheOREM 6 The problem $(\mathcal{P})$ admits a unique solution $u$ in $W(0, T ; V)$.

Let $u$ be the solution of problem $(\mathcal{P})$. Then $u \in L^{2}(0, T ; V) \cap L^{2}(0, T ; H)$ and we can consider the proper orthogonal decomposition of $u$ in both cases $X=V$ and $X=H$. Let $\left(\psi_{k}\right)_{k \geq 1}$ be a set of POD eigenvectors associated with $u$ in one of both cases $X=V$ or $X=H$. Let $\ell \geq 1$ be an integer and let $Y_{\ell}=\operatorname{span}\left(\psi_{1}, \cdots, \psi_{\ell}\right)$ be the approximated subspace of order $\ell$. We consider the following problem ;

$$
\left(\mathcal{P}_{\ell}\right)\left\{\begin{aligned}
\frac{d}{d t}\left(U_{\ell}(t), \varphi\right)_{H}+a\left(U_{\ell}(t), \varphi\right) & =\langle f(t), \varphi\rangle_{V^{\prime} \times V} & & t \in[0, T], \varphi \in Y_{\ell} \\
\left(U_{\ell}(0), \varphi\right)_{H} & =(\phi, \varphi)_{H} & & \varphi \in Y_{\ell}
\end{aligned}\right.
$$

which admits a unique solution $U_{\ell} \in C\left(0, T ; Y_{\ell}\right)$. The following theorem is now well known [6]:

TheOREM 7 In both cases $X=V$ and $X=H$, the sequence $\left(U_{\ell}\right)_{\ell \geq 1}$ of the solutions of the problems $\left(\mathcal{P}_{\ell}\right)$ converges towards the solution $u$ of the problem $(\mathcal{P})$ as $\ell \rightarrow+\infty$ in $L^{2}(0, T ; V)$ strong.

\section{Estimates of the Error of POD-Approximation in a Regular Case}

Now we want to give an estimate of the error $\left\|U_{\ell}-u\right\|_{L^{2}(0, T ; V)}$. We consider a case when the function $u$ is regular. We make the following assumption: 
Assumption 1. Still denoting $u$ the solution of the problem $(\mathcal{P})$ in $W(0, T ; V)$, we assume that the time derivative $d u / d t$ is in the space $L^{2}(0, T ; V)$.

This assumption is sensible because of the following result ([12] theorem 3.2 , p. 70$)$ :

THEOREM 8 If $f$ and $d f / d t$ are in $L^{2}(0, T ; H)$ and if the initial condition $\phi$ is in $V$, then $d u / d t$ is in $L^{2}(0, T ; V)$.

Under assumption 1, we can also consider the POD approximation of the problem $(\mathcal{P})$ by defining the proper orthogonal decomposition associated with $(u, d u / d t)$. In this case we will obtain an estimate of the error of approximation according to the POD eigenvalues.

\subsection{Case of the POD Associated with $u$}

We consider the case $X=V$ and we still denote $\left(\psi_{k}\right)_{k>1}$ a sequence of POD eigenvectors associated with $u$. The theorem 4 allows us to write the following equality in $V$ for $t \in[0, T]$ and for any $\ell \geq 1$ :

$$
u(t)=\sum_{k=1}^{\ell}\left(u(t), \psi_{k}\right)_{V} \psi_{k}+\sum_{k=\ell+1}^{\infty}\left(u(t), \psi_{k}\right)_{V} \psi_{k}
$$

We define two fonctions $u_{\ell}$ and $\widetilde{u}_{\ell}$ by posing for $t \in[0, T]$ :

$$
u_{\ell}(t)=\sum_{k=1}^{\ell}\left(u(t), \psi_{k}\right)_{V} \psi_{k}, \quad \tilde{u}_{\ell}(t)=\sum_{k=\ell+1}^{\infty}\left(u(t), \psi_{k}\right)_{V} \psi_{k} .
$$

As the function $U_{\ell}$, which is the solution of the problem $\left(\mathcal{P}_{\ell}\right)$, is in the space $Y_{\ell}=\operatorname{span}\left(\psi_{1}, \cdots, \psi_{\ell}\right)$, we have $U_{\ell}(t)=\sum_{k=1}^{\ell}\left(U_{\ell}(t), \psi_{k}\right)_{V} \psi_{k}$. By definition, the set $\left(\psi_{k}\right)_{k \geq 1}$ is orthonormal in $V$, so the Pythagore theorem gives:

$$
\left\|u-U_{\ell}\right\|_{L^{2}(0, T ; V)}^{2}=\left\|u_{\ell}-U_{\ell}\right\|_{L^{2}(0, T ; V)}^{2}+\left\|\widetilde{u}_{\ell}\right\|_{L^{2}(0, T ; V)}^{2} .
$$

We observe the following equality:

$$
\left\|\widetilde{u}_{\ell}\right\|_{L^{2}(0, T ; V)}^{2}=T \sum_{k=\ell+1}^{\infty}\left(K \psi_{k}, \psi_{k}\right)_{V}=T \sum_{k=\ell+1}^{\infty} \lambda_{k},
$$

and we know from theorem 4 that the rest $\sum_{k=\ell+1}^{\infty} \lambda_{k}$ tends towards zero as $\ell \rightarrow \infty$. We only have to estimate the term $\left\|u_{\ell}-U_{\ell}\right\|_{L^{2}(0, T ; V)}^{2}$. We denote $z_{\ell}=U_{\ell}-u_{\ell}$. The function $u$ (resp. $U_{\ell}$ ) is the solution 
of problem $(\mathcal{P})$ (resp. $\left(\mathcal{P}_{\ell}\right)$ ) so the function $z_{\ell}$ satisfies the following equality for $\varphi \in Y_{\ell}$ :

$$
\frac{d}{d t}\left(z_{\ell}(t), \varphi\right)_{H}+a\left(z_{\ell}(t), \varphi\right)=\frac{d}{d t}\left(\widetilde{u}_{\ell}(t), \varphi\right)_{H}+a\left(\widetilde{u}_{\ell}(t), \varphi\right), t \in[0, T],
$$

as well as $\left(z_{\ell}(0), \varphi\right)_{H}=\left(\widetilde{u}_{\ell}(0), \varphi\right)_{H}$. If we take $\varphi=z_{\ell}(0)$, we obtain $\left\|z_{\ell}(0)\right\|_{H} \leq\left\|\widetilde{u}_{\ell}(0)\right\|_{H}$ and if we take $\varphi=z_{\ell}(t)$ in equality (14), we obtain the following equality after integration on $[0, T]$ :

$$
\begin{aligned}
& \frac{1}{2}\left\|z_{\ell}(T)\right\|_{H}^{2}+\int_{0}^{T} a\left(z_{\ell}(t), z_{\ell}(t)\right) d t \\
= & \int_{0}^{T}\left(\frac{d \widetilde{u}_{\ell}}{d t}(t), z_{\ell}(t)\right)_{H} d t+\int_{0}^{T} a\left(\widetilde{u}_{\ell}(t), z_{\ell}(t)\right) d t+\frac{1}{2}\left\|z_{\ell}(0)\right\|_{H}^{2} .
\end{aligned}
$$

Then we get:

$$
\begin{aligned}
\kappa\left\|z_{\ell}\right\|_{L^{2}(0, T ; V)}^{2} \leq & \frac{\varepsilon}{2}\left\|\frac{d \widetilde{u}_{\ell}}{d t}\right\|_{L^{2}(0, T ; H)}^{2}+\left(\frac{\alpha}{2 \varepsilon}+\frac{\beta}{2}\right)\left\|z_{\ell}\right\|_{L^{2}(0, T ; V)}^{2} \\
& +\frac{\beta}{2}\left\|\tilde{u}_{\ell}\right\|_{L^{2}(0, T ; V)}^{2}+\frac{1}{2}\left\|\tilde{u}_{\ell}(0)\right\|_{H}^{2},
\end{aligned}
$$

where $\varepsilon>0$ is a real to be chosen below. Let us recall that the definition of the form $a$ gives for any $\varphi \in V: \kappa\|\varphi\|_{V}^{2} \leq a(\varphi, \varphi) \leq \beta\|\varphi\|_{V}^{2}$. Moreover we assume $\kappa-\beta / 2>0$. Now we choose $\varepsilon>0$ such that $\kappa-\alpha / 2 \varepsilon-\beta / 2>0$ and we obtain the following estimate:

$$
\left(\kappa-\frac{\alpha}{2 \varepsilon}-\frac{\beta}{2}\right)\left\|z_{\ell}\right\|_{L^{2}(0, T ; V)}^{2} \leq \frac{\varepsilon}{2}\left\|\frac{d \widetilde{u}_{\ell}}{d t}\right\|_{L^{2}(0, T ; H)}^{2}+\frac{\beta}{2}\left\|\widetilde{u}_{\ell}\right\|_{L^{2}(0, T ; V)}^{2}+\frac{1}{2}\left\|\widetilde{u}_{\ell}(0)\right\|_{H}^{2}
$$

The term $\left\|\tilde{u}_{\ell}\right\|_{L^{2}(0, T ; V)}^{2}$ tends towards zero as $\ell \rightarrow \infty$ by definition of $\widetilde{u}_{\ell}$ and because of equality (6) in theorem 4 . Moreover equality (10) holds in $V$ for almost any $t \in[0, T]$, so in $H$ for any $t \in[0, T]$ because $u \in C(0, T ; H)$, in particular for $t=0$, and we obtain that the term $\left\|\widetilde{u}_{\ell}(0)\right\|_{H}^{2}$ tends towards zero as $\ell \rightarrow \infty$. However we have in general $d u / d t \in L^{2}\left(0, T ; V^{\prime}\right)$, which does not ensure the convergence of the term $\left\|d \widetilde{u}_{\ell} / d t\right\|_{L^{2}(0, T ; H)}^{2}$. That is why we are led to make assumption 1.

THEOREM 9 Under assumption 1 , we choose $\varepsilon>0$ such that $\kappa-\alpha / 2 \varepsilon-$ $\beta / 2>0$ and we set:

$$
\rho_{\ell}=\frac{1}{\kappa-\alpha / 2 \varepsilon-\beta / 2}\left(\frac{\varepsilon}{2}\left\|\frac{d \widetilde{u}_{\ell}}{d t}\right\|_{L^{2}(0, T ; H)}^{2}+\frac{1}{2}\left\|\widetilde{u}_{\ell}(0)\right\|_{H}^{2}\right) .
$$

We choose $X=V$ and we consider the proper orthogonal decomposition associated to the function $u$. We get the following estimate:

$$
\left\|u-U_{\ell}\right\|_{L^{2}(0, T ; V)}^{2} \leq \rho_{\ell}+\frac{(\kappa-\alpha / 2 \varepsilon) T}{\kappa-\alpha / 2 \varepsilon-\beta / 2} \sum_{k=\ell+1}^{\infty} \lambda_{k} \underset{\ell \rightarrow \infty}{\longrightarrow} 0,
$$


on the error between the solution $u$ of the problem $(\mathcal{P})$ and the approximation $U_{\ell}$, which is the solution of the problem $\left(\mathcal{P}_{\ell}\right)$.

Proof. According to inequality (17), it suffices to proove that the term $\left\|d \widetilde{u}_{\ell} / d t\right\|_{L^{2}(0, T ; H)}^{2}$ tends towards zero as $\ell \rightarrow \infty$. As the subspace $Y=\overline{\operatorname{span}\left(\psi_{k}\right)_{k \geq 1}} V$ is closed in $V$ and as $u \in L^{2}(0, T ; Y)$ according to equality (10), we obtain, under assumption 1 , that $d u / d t \in$ $L^{2}(0, T ; Y)$. We can then write the following equality in $V$ for $t \in[0, T]$ : $d u / d t(t)=\sum_{k=1}^{\infty}\left(d u / d t(t), \psi_{k}\right)_{V} \psi_{k}$. Then we have $\left\|d \widetilde{u}_{\ell} / d t\right\|_{L^{2}(0, T ; V)}^{2}=$ $\left\|\sum_{k=\ell+1}^{\infty}\left(d u / d t(t), \psi_{k}\right)_{V} \psi_{k}\right\|_{L^{2}(0, T ; V)}^{2}$ which tends towards zero as $\ell \rightarrow$ $\infty$. On the other hand, we have the bound $\|\cdot\|_{H} \leq \alpha\left\|_{\|}\right\|_{V}$ and we get the expected result from equalities (12) and (13) as well as from inequality (17).

\subsection{Case of the POD Associated with $(u, d u / d t)$}

The definition of the term $\rho_{\ell}$ in theorem 9 let the term

$$
\left\|d \widetilde{u}_{\ell} / d t\right\|_{L^{2}(0, T ; H)}^{2}
$$

arise, which represents the energy of the rest of the time derivative of $u$. It seems quite natural to consider the proper orthogonal decomposition associated with the set $(u, d u / d t)$ so that the POD eigenvalues also take into account the energy of $d u / d t$.

We still consider the case $X=V$ and we denote $\left(\psi_{k}\right)_{k \geq 1}$ a set of POD eigenvectors associated with $(u, d u / d t)$. In this case we have the following equality, according to theorem 4 , in particular according to equality (6):

$$
\sum_{k=\ell+1}^{\infty} \int_{0}^{T}\left(\frac{d u}{d t}(t), \psi_{k}\right)_{V}^{2} d t+\sum_{k=\ell+1}^{\infty} \int_{0}^{T}\left(u(t), \psi_{k}\right)_{V}^{2} d t=T \sum_{k=\ell+1}^{\infty} \lambda_{k}
$$

We set $c=\max \left(\varepsilon \alpha^{2} /(2 \kappa-\alpha / \varepsilon-\beta),(\kappa-\alpha / 2 \varepsilon) /(\kappa-\alpha / 2 \varepsilon-\beta / 2)\right)$ and we can now express the following theorem:

THEOREM 10 Under assumption 1 , we choose $X=V$ and we consider the proper orthogonal decomposition associated with $(u, d u / d t)$. With the above notations, we obtain the following estimate:

$$
\left\|u-U_{\ell}\right\|_{L^{2}(0, T ; V)}^{2} \leq c T \sum_{k=\ell+1}^{\infty} \lambda_{k}+\frac{1}{2 \kappa-\alpha / \varepsilon-\beta}\left\|\widetilde{u}_{\ell}(0)\right\|_{H}^{2} \underset{\ell \rightarrow \infty}{\longrightarrow} 0 .
$$




\section{Choosing the Order of Approximation}

When proper orthogonal decomposition is utilized for model reduction, the question arises of the choice of the order $\ell \geq 1$ of approximation. The integer $\ell$ must be large enough for the approximation to be good and small enough for the model to be reduced enough. The usual criterion (cf. for example $[1,5,8,9,10,11]$ ) consists in setting a percentage $\delta \in[0,1]$ then in choosing the smaller integer $\ell \geq 1$ such that:

$$
\sum_{k=1}^{\ell} \lambda_{k} / \sum_{k=1}^{\infty} \lambda_{k} \geq \delta,
$$

where the $\lambda_{k}$ s are the POD eigenvalues. As the sum of the POD eigenvalues is equal to the energy of $u$, this criterion consists in projecting the studied system onto the modes which capture the largest portion of the energy of $u$. We are going to see that this criterion can be irrelevant and we will propose alternative ideas.

\subsection{A Counter-example to the Usual Criterion of the Choice of the Order of Approximation}

We still consider the parabolic case of the problem $(\mathcal{P})$. We set $\Omega=$ ]0, $1\left[, V=H_{0}^{1}(\Omega), H=L^{2}(\Omega)\right.$ and $X=V$. We define the bilinear form $a$ by posing $a(.,)=.(., .)_{V}$ so that the problem $(\mathcal{P})$ is the variationnal form of the heat equation with homogeneous Dirichlet boundary conditions. Let $\psi_{1}$ and $\psi_{2}$ be orthonormal vectors in $V$ but not in $H$ defined by:

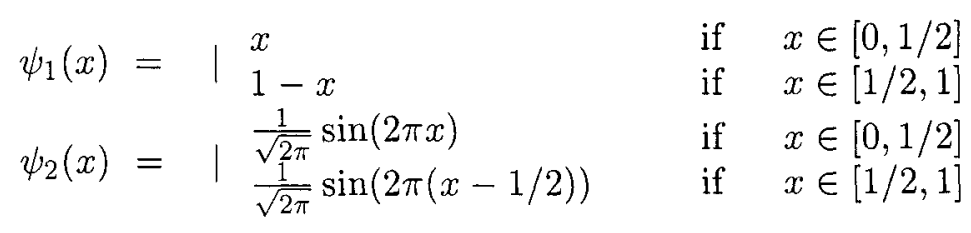

Then we have

$$
\left\|\psi_{1}\right\|_{H}^{2}=1 / 12,\left\|\psi_{2}\right\|_{H}^{2}=1 /\left(4 \pi^{2}\right) \text { and }\left(\psi_{1}, \psi_{2}\right)_{H}=(2 \pi)^{-3 / 2}
$$

We set $T=1$ and we define two orthogonal functions $h_{1}$ and $h_{2}$ in $L^{2}(0, T)$ by posing for $t \in[0, T]: h_{1}(t)=1$ and $h_{2}(t)=1.2 \sin (20 \pi t)$. We set at last for $x \in \Omega$ and $t \in[0, T]: u(x, t)=h_{1}(t) \psi_{1}(x)+$ $h_{2}(t) \psi_{2}(x)$. We assume that the function $u$ is the solution of the problem $(\mathcal{P})$ for a well chosen right-hand term $f$. We still denote $K$ the POD operator associated with $u$ and we observe that $K \psi_{1}=\psi_{1}$ and $K \psi_{2}=$ $0.72 \psi_{2}$ so the vectors $\left(\psi_{1}, \psi_{2}\right)$ are the POD eigenvectors associated with $u$ and the POD eigenvalues are $\lambda_{1}=1$ and $\lambda_{2}=0.72$. We denote $y_{1}$ 
(resp. $\left.y_{2}\right)$ the solution of the projection of the problem $(\mathcal{P})$ onto $\operatorname{span}\left(\psi_{1}\right)$ $\left(\right.$ resp. $\left.\operatorname{span}\left(\psi_{2}\right)\right)$. As $\lambda_{1}>\lambda_{2}$ we expect the projection onto $\operatorname{span}\left(\psi_{1}\right)$ to be better than that onto $\operatorname{span}\left(\psi_{2}\right)$, that is:

$$
\left\|u-y_{1}\right\|_{L^{2}(0, T ; V)} \leq\left\|u-y_{2}\right\|_{L^{2}(0, T ; V)} .
$$

In fact, this inequality is not satisfied. Indeed a computation with Maple allows us to obtain:

$$
\left\|u-y_{1}\right\|_{L^{2}(0, T ; V)}^{2} \simeq 1.2641, \quad\left\|u-y_{2}\right\|_{L^{2}(0, T ; V)}^{2}=1+\left(1-e^{-8 \pi^{2}}\right) / 4 \pi,
$$

that is:

$$
\left\|u-y_{1}\right\|_{L^{2}(0, T ; V)}>\left\|u-y_{2}\right\|_{L^{2}(0, T ; V)},
$$

instead of the expected inequality (24). If we set the percentage $\delta=58 \%$ and if we apply criterion (22), we find $\ell=1$ and we compute $y_{1}$, whereas the function $y_{2}$ is a best approximation of the solution $u$ of the problem $(\mathcal{P})$. We could conclude that the POD set $\left(\psi_{1}, \psi_{2}\right)$ is not well indexed and that the indexation must be modified to consider the set $\left(\psi_{2}, \psi_{1}\right)$. However, if the number of POD eigenvectors is infinite, it is difficult to reindex them. We rather consider the point of view which consists in keeping the same order of indexation as that of the eigenvalues and in going deeper into the calculation of the approximation.

\subsection{Definition of Some Criteria for Choosing the Order of Approximation}

Let us first mention two natural criteria for choosing the order of approximation. The first natural criterion is the absolute value $\ell \geq 1$ of the order of approximation: we can decide not to go over a certain value, for instance in order to limit the computation time. This is not the usual point of view: in general, one prefers to choose the smallest integer $\ell \geq 1$ which satisfies a certain condition, for example the inequality (22), which amounts to favouring the precision of the approximation rather than the speed of computation; we will follow this line by defining criteria for the order of approximation. The second natural criterion consists in utilizing the bounds (19) and (21) in theorems 9 and 10 to ensure a given precision.

We still make the regularity assumption 1 and we set $X=V$. We consider the case of the proper orthogonal decomposition associated with the solution $u$ of the problem $(\mathcal{P})$. We propose the following definition:

Definition 11 We define the real numbers $\varepsilon, \rho_{\ell}>0$ as theorem 9 . The integer $\ell \geq 1$ is a good order of approximation if we have the following 

inequality:

$$
\rho_{\ell}+\frac{(\kappa-\alpha / 2 \varepsilon) T}{\kappa-\alpha / 2 \varepsilon-\beta / 2} \sum_{k=\ell+1}^{\infty} \lambda_{k} \leq T \sum_{k=1}^{\ell} \lambda_{k} .
$$

THEOREM 12 If the integer $\ell \geq 1$ is a good order of approximation, the Galerkin projection of the problem $(\mathcal{P})$ onto the subspace $Y_{\ell}$ is better than the Galerkin projection onto the subspace $Y_{\ell}^{\varphi}$, for any subspace $Y_{\ell}^{\varphi}$ orthogonal to $Y_{\ell}$ in $Y$, in the following sense:

$$
\left\|u-U_{\ell}\right\|_{L^{2}(0, T ; V)} \leq\left\|u-U_{\ell}^{\varphi}\right\|_{L^{2}(0, T ; V)}
$$

where $U_{\ell}^{\varphi}$ is the solution obtained by Galerkin projection onto $Y_{\ell}^{\varphi}$.

Definition 13 We define real numbers $\varepsilon, \rho_{\ell}>0$ as in theorem 9 . The integer $\ell \geq 1$ is a very good order of approximation if the following inequality holds:

$$
\rho_{\ell}+\frac{(\kappa-\alpha / 2 \varepsilon) T}{\kappa-\alpha / 2 \varepsilon-\beta / 2} \sum_{k=\ell+1}^{\infty} \lambda_{k} \leq T \lambda_{\ell} .
$$

THEOREM 14 If the integer $\ell \geq 1$ is a very good order of approximation, then for any integer $k \geq \ell+1$ the Galerkin projection of the problem $(\mathcal{P})$ onto the subspace $Y_{\ell}=\operatorname{span}\left(\psi_{1}, \cdots, \psi_{\ell-1}, \psi_{\ell}\right)$ is better in the sense of the norm $L^{2}(0, T ; V)$ than the projection onto the subspace $\operatorname{span}\left(\psi_{1}, \cdots, \psi_{\ell-1}, \psi_{k}\right)$.

Analogous definitions can be expessed in the case of the POD associated with $(u, d u / d t)$.

\section{Conclusion}

We have recalled the principle of proper orthogonal decomposition and we have considered POD-Galerkin methods for a parabolic problem. If the solution $u$ of the parabolic problem $(\mathcal{P})$ is regular, i.e. $u$ satisfies assumption 1, we can obtain bounds of the error of the POD-Galerkin approximation in the case $X=V$. We have considered the POD associated with $u$ and the POD associated with $(u, d u / d t)$. These bounds do not depend on the discretization scheme and allow us to define some criteria for choosing the order of approximation.

\section{References}

[1] H. Banks, L. Joyner, B. Wincheski, and W. Winfree. Nondestructive evaluation using a reduced order computational methodology, 2000. Nasa/CR-2000-209870, ICASE Report No. 2000-10. 
[2] G. Berkooz, P. Holmes, and J. Lumley. The proper orthogonal decomposition in the analysis of turbulent flows. Annu. Rev. Fluid Mech., 25:539-575, 1993.

[3] J. Bonnet, L. Cordier, J. Delville, M. Glauser, and L. Ukeiley. Examination of large-scale structures in a turbulent plane mixing layer. Part 1. Proper orthogonal decomposition. J. Fluid Mech., 391:91-122, 1999.

[4] R. Dautray and J.L. Lions. Analyse mathématique et calcul numérique pour les sciences et les techniques, tome 3. Masson, Paris, 1985.

[5] A. Glezer, Z. Kadioglu, and A. Pearlstein. Development of an extended proper orthogonal decomposition and its application to a time periodically forced plane mixing layer. Phys. Fluids A, Vol. 1, No. 8:1363-1373, 1989.

[6] T. Henri and J.-P. Yvon. Stability of the POD and convergence of the PODGalerkin method for parabolic problems, 2002. preprint IRMAR 02-40.

[7] K. Kunisch and S. Volkwein. Galerkin proper orthogonal decomposition methods for parabolic problems. Numer. Math., 90:117-148, 2001.

[8] S. Lall, J.E. Marsden, and S. Glavaski. Empirical model reduction of controlled nonlinear systems, 1999. Proceedings of the IFAC World Congress.

[9] B.C. Moore. Principal component analysis in linear systems : controllability, observability and model reduction. IEEE Transactions on Automatic Control, vol. AC-26, no 1, 1981.

[10] S.S. Ravindran. Proper orthogonal decomposition in optimal control of fluids, 1999. Nasa/TM-1999-209113.

[11] S.Y. Shvartsman, C. Theodoropoulos, R. Rico-Martinez, I.G. Kevrekidis, E.S. Titi, and T.J. Mountziaris. Order reduction for nonlinear dynamic models of distributed reacting systems. J. of Process Control, 10:177-184, 2000.

[12] R. Temam. Infinite-dimensional dynamical systems in mechanics and physics. Spinger-Verlag, New York, 1988. 